\title{
Sobre dos Teoremas Combinatorios
}

\author{
Moisés Samuel Toledo Julián ${ }^{1}$, Alex Molina Sotomayor ${ }^{2}$, Napoleón Caro Tuesta $^{3}$
}

Resumen: Presentamos dos teoremas importantes en la topología algebraica combinatoria y la geometría combinatoria convexa, estos son el teorema del nervio y el teorema de Helly, dando ejemplos de su uso y relevancia. Mostramos que extensores absolutos son equivalentes a retractos absolutos y que son propiedades topológicas lo cual permite, por ejemplo, obtener triangulaciones para espacios topológicos expresados en términos del nervio del complejo simplicial asociado. Así también la estructuras convexas abstractas tienen principal relevancia para espacios metrizables, en particular los conjuntos convexos son extensores absolutos y por tanto retractos, pudiendo así obtenerse cubrimientos regulares y buenos cubrimientos. El patrón de intersección de estos cubrimientos por convexos da lugar a tres números combinatorios importantes, el número de Helly, Radon y Caratheodory. Culminamos haciendo evidente algunas propiedades combinatorias que poseen estos números, en particular que entre los diversos usos del número de Helly.

Palabras clave: estructura convexa, nervio, retracto absoluto, $d$-representabilidad.

\section{About two Combinatorial Theorems}

\begin{abstract}
We present two important theorems in combinatorial algebraic topology and convex combinatorial geometry, these are the nerve theorem and Helly's theorem, giving examples of their use and relevance. We show that absolute extenders are equivalent to absolute retractions and that they are topological properties which allows, for example, to obtain triangulations for topological spaces expressed in terms of the rib of the associated simplicial complex. Thus also the abstract convex structures have main relevance for metrizable spaces, in particular the convex sets are absolute extensors and therefore retracted, thus being able to obtain regular coverings and good coverings. The intersection pattern of these coverings by convex gives rise to three important combinatorial numbers, the Helly number, Radon and Caratheodory. We conclude by making evident some combinatorial properties that these numbers possess, in particular that among the various uses of the Helly number.
\end{abstract}

Keywords: convex structure, nerve, absolute retraction, $d$-representability.

Recibido: 17/02/2021.

Aceptado: 10/06/2021.

Publicado on line: 30/06/2021.

(C) Los autores. Este artículo es publicado por la Revista PESQUIMAT de la Facultad de Ciencias Matemáticas, Universidad Nacional Mayor de San Marcos. Este es un artículo de acceso abierto, distribuido bajo los términos de la licencia Creative Commons Atribución-No Comercial-Compartir Igual 4.0 Internacional.(http://creativecommons.org/licenses/by-nc-sa/4.0/) que permite el uso no comercial, distribución y reproducción en cualquier medio, siempre que la obra original sea debidamente citada. Para uso comercial, por favor póngase en contacto con revistapesquimat.matematica@unmsm.edu.pe

\footnotetext{
${ }^{1}$ UNMSM, Facultad de Ciencias Matemáticas. e-mail: moises.toledo@unmsm.edu.pe

${ }^{2}$ UNMSM, Facultad de Ciencias Matemáticas, e-mail: alexcantuta@gmail.com

${ }^{3}$ UFPB, Departamento de Matemática, e-mail: napo.caro@gmail.com
} 


\section{Introducción}

Una eficiente manera de investigar propiedades de un espacio topológico es dividirlo en piezas y examinar cómo ellas están pegadas. Dividir un espacio topológico general con una estructura similar a una triangulación, siempre que esto sea posible, permite emplear una analogía con resultados que garantizan propiedades homotópicas y homológicas simples. Por ejemplo, Borsuk [1] muestra que si un espacio compacto finito dimensional $C$ posee una descomposición como suma finita de retractos absolutos tal que cualquier intersección no vacía de estos retractos es también un retracto absoluto, resulta que todos los grupos de homotopía y homología de $C$ son determinados por las propiedades combinatorias de la descomposición.

En adelante, la palabra espacio denotará un espacio topológico, y por una aplicación se entenderá una aplicación continua. Para dividir un espacio que pertenece a una clase $\mathfrak{C}$ de espacios topológicos, debemos especificar qué es una pieza, esta debe ser lo más parecido a un símplice.

Definición 1.1. Un espacio $Y$ es un extensor absoluto para un espacio $X$ si cada aplicación de un subconjunto cerrado de $X$ hacia $Y$ se extiende sobre el espacio entero $X$. La clase de extensores absolutos de todos los espacios de la clase $\mathfrak{C}$ es denotado por EA( $\mathfrak{C})$. Escribiremos $\mathrm{EA}(X)$ para abreviar $\mathrm{EA}(\{X\})$.

Una clase $\mathfrak{C}$ es un conjunto de espacios topológicos débilmente hereditarios (DH), si se cumple

(DH-1) $\mathfrak{C}$ es topológico, es decir, si $\mathfrak{C}$ contiene un espacio $X$ entonces contiene a cualquier imagen homeomorfa a $X$

(DH-2) $\mathfrak{C}$ es débilmente hereditario, es decir, si $\mathfrak{C}$ contiene un espacio $X$ entonces contiene a cualquier subespacio cerrado de $X$

Ejemplo 1.1. Algunas clases $\mathfrak{C}$ de espacios topológicos débilmente hereditarios son: $\mathcal{H}$ Hausdorff, $\mathcal{R}$ regulares, $\mathcal{C} \mathcal{R}$ completamente regulares, $\mathcal{M}$ metrizables, $\mathcal{C}_{1}$ 1-numerable, $\mathcal{C}_{2}$ 2-numerable, $\mathcal{N}$ normales, $\mathcal{P}$ perfectamente normales, $\mathcal{F}$ totalmente normales, $\mathcal{K}$ compactos, $\mathcal{L} \mathcal{K}$ localmente compactos, $\mathcal{L}$ Lindelof.

Dado que la intersección de dos clases cualesquiera de espacios topológicos débilmente hereditarios es también una clase topológica débilmente hereditaria, obtenemos espacios como: $\mathcal{K} \mathcal{M}$ espacios compactos metrizables, $\mathcal{S M}$ espacios separables metrizables. Así también, la unión de dos cualesquiera espacios débilmente hereditarios es también débilmente hereditario.

Definición 1.2. Un espacio $Y$ es un extensor de vecindad absoluto de un espacio $X$ si para cada subespacio cerrado $A$ de $X$ la aplicación $f: A \rightarrow Y$ puede ser extendida sobre algún subespacio abierto $U$ de $X$ que contiene $A$. La clase de extensores de vecindad absoluta de todos los espacios de clase $\mathfrak{C}$ es denotado por $\operatorname{EVA}(\mathfrak{C})$.

Considerando la clase de espacios topológicos normales $\mathcal{N}$, se tiene el siguiente resultado

Teorema 1 (ver [5] pp.46). Si un espacio $Y$ es la unión finita de subconjuntos abiertos tales que cada uno es un $\operatorname{EVA}(\mathcal{N})$ entonces $Y$ es un $\operatorname{EVA}(\mathcal{N})$.

Definimos los cubrimientos regulares que permitirán dotar a un espacio de estructuras similares a triangulaciones.

Definición 1.3. Sea $\mathfrak{C}$ una clase de espacios topológicos. Un cubrimiento es un $\mathfrak{C}$-cubrimiento si la intersección de cualquier colección no vacía, de elementos del cubrimiento, pertenece a $\mathfrak{C}$. Un $\mathrm{EA}(\mathfrak{C})$-cubrimiento localmente finito que es cerrado y localmente de dimensión finita o abierto es denominado regular para la clase $\mathfrak{C}$.

Ejemplo 1.2. Cubrimientos localmente finitos de un espacio euclidiano por bolas abiertas y cubrimientos de un complejo simplicial finito por sus símplices son casos de cubrimientos regulares. 


\section{Metodología}

\subsection{Nervios, cubrimientos y complejos simpliciales}

\section{Nervio de un cubrimiento}

Un cubrimiento abierto de un espacio topológico $X$ es una colección $\mathcal{U}=\left\{U_{i}\right\}$ de subconjuntos abiertos $U_{i} \subseteq X, i \in I$ donde $I$ es un conjunto de índices, tal que $X=\bigcup_{i \in I} U_{i}$.

Dado un cubrimiento $\mathcal{U}=\left\{U_{i}\right\}$ de un espacio topológico $X$, el nervio del cubrimiento es el complejo simplicial abstracto $\mathcal{N}(\mathcal{U})$ cuyo conjunto de vértices es $\mathcal{U}$ y tal que

$$
\sigma=\left[U_{i_{0}}, U_{i_{1}}, \ldots, U_{i_{k}}\right] \in \mathcal{N}(\mathcal{U}) \Leftrightarrow \bigcap_{j=0}^{k} U_{i_{j}} \neq \emptyset
$$

Ejemplo 2.1. Consideremos el espacio $X$ y el cubrimiento $\mathcal{U}=\left\{U_{i}\right\}$, tal como se indica en la figura, obtenemos el nervio $\mathcal{N}(\mathcal{U})$ a partir de las intersecciones no vacías entre los $U_{i}$
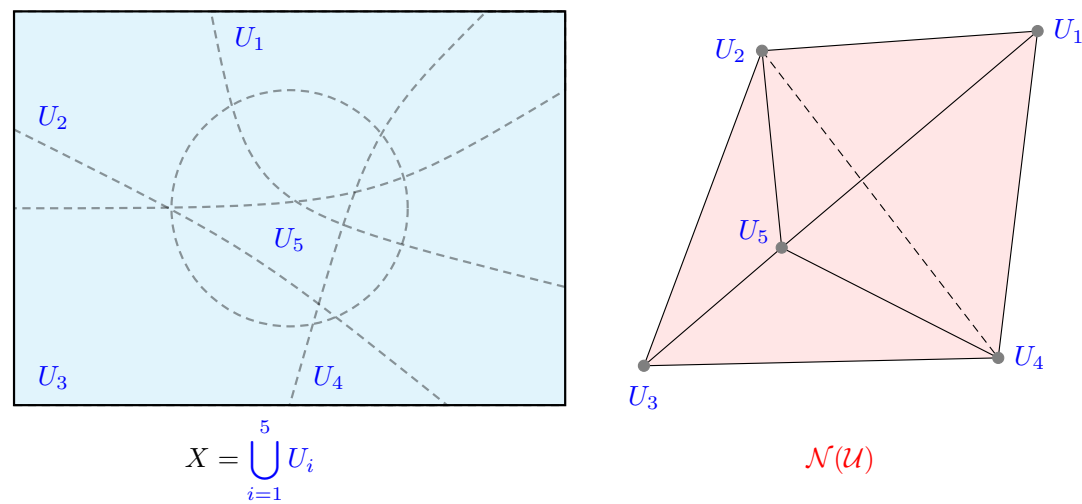

\section{Nervio de un complejo simplicial}

Se dice que una colección finita de conjuntos $\mathcal{F}=\left\{F_{1}, F_{2}, \ldots, F_{s}\right\}$ y el conjunto de vértices $a_{1}, a_{2}, \ldots, a_{s}$ de un complejo simplicial $K$ están relacionados entre si de tal manera que la subcolección $F_{i_{0}}, F_{i_{1}}, \ldots, F_{i_{r}}$, posee intersección no vacía si y solo si los vértices $a_{i_{0}}, a_{i_{1}}, \ldots, a_{i_{r}}$ pertenecen a un símplice de $K$. Al complejo $K$ se le denomina el nervio de la colección $\mathcal{F}$, en símbolos $K=\mathcal{N}(\mathcal{F})$.

Una cara de un complejo simplicial $K$ es denominada una careta, símplice principal o símplice maximal si no está propiamente contenido en ninguna otra cara de $K$. El nervio $\mathcal{N}(K)$ de un complejo simplicial $K$, es también un complejo simplicial, definido por

- los vértices de $\mathcal{N}(K)$ están en correspondencia uno a uno con las caretas de $K$

- un conjunto de vértices de $\mathcal{N}(K)$ determina un símplice de $\mathcal{N}(K)$ si y solamente si las caretas correspondientes de $K$ tienen intersección no vacía

Una cara $M$ de un complejo simplicial $K$ es denominada básica si $M \neq \emptyset$ y coincide con la intersección de todas las caretas de $K$ que poseen intersección no vacía con $M$. Es decir, una cara $M \neq \emptyset$ es básica si para cada careta $F$ de $K, F \cap M \neq \emptyset$ implica $F \supset M$ y $M=\bigcap F$, la intersección es sobre todas las caretas $F$ de $K$ que contienen a $M$. Naturalmente, diferentes caras básicas son disjuntas, y cada careta de $K$ contiene al menos una cara básica de $K$.

Sea $K$ un complejo simplicial y $M$ una cara básica de $K$. Sea $F_{i}, i=1, \ldots, p$, todas las caretas de $K$ conteniendo $M$, esto es $M=\bigcap_{i=1}^{p} F_{i}$, y cualquier otra careta de $K$ es disjunta de $M$. Sea $v_{i}=v\left(F_{i}\right)$ el vértice de $\mathcal{N}(K)$ que corresponde a $F_{i}$. Desde que $M \neq \emptyset$, el símplice $\left[v_{1}, \ldots, v_{p}\right]$ con vértices $v_{1}, \ldots, v_{p}$ es una cara de $\mathcal{N}(K)$, denotado por $\bar{M}$. 


\section{Resultados y discusión}

\subsection{Aplicaciones en la topología algebraica}

\section{Caracterización de espacios mediante complejos simpliciales y nervios}

Lema 1. $\bar{M}$ es una careta de $\mathcal{N}(K)$.

Demostración. Si $\bar{M}$ no es una careta, existe en $\mathcal{N}(K)$ una cara $N=\{v\} \cup \bar{M}$ con $v \notin \bar{M}$. El vértice $v$ corresponde a alguna careta $F$ de $K$, diferente a los $F_{1}, \ldots, F_{p}$. Luego $F \cap M=\emptyset$, por otro lado, dado que $N \in \mathcal{N}(K)$, se tiene $\emptyset \neq F \cap \bigcap_{i=1}^{p} F_{i}=F \cap M$, lo cual es una contradicción. Por tanto $\bar{M}$ es una careta de $\mathcal{N}(K)$.

De este modo, a cada cara básica $M$ de $K$ le corresponde una careta $\bar{M}$ de $\mathcal{N}(K)$.

Lema 2. Cada careta $N$ de $\mathcal{N}(K)$ es $\bar{M}$ para una cara básica conveniente $M$ de $K$.

Demostración. Sean $v_{1}, \ldots, v_{r}$ los vértices de $N$ y $F_{1}, \ldots, F_{r}$ las correspondientes caretas de $K$. Entonces $M=\bigcap_{i=1}^{r} F_{i} \neq \emptyset$ y cada careta $F \notin\left\{F_{1}, \ldots, F_{r}\right\}$ de $K$ debe estar fuera de $M$, pues si $F \cap \bigcap_{i=1}^{r} F_{i} \neq \emptyset$ entonces $N \cup v(F) \in \mathcal{N}(K)$, contradiciendo la maximalidad de $N$. Así, $M$ es una cara básica de $K$ y $N=\bar{M}$.

Del lema 1 y 2 se tiene una correspondencia uno a uno entre las caras básicas de $K$ y las caretas de $\mathcal{N}(K)$. De modo que una careta $F$ de $K$ contiene una cara básica $M$ de $K$ si y solo si el vértice $v(F)$ de $\mathcal{N}(K)$ pertenece a la careta $\bar{M}$ de $\mathcal{N}(K)$.

El siguiente resultado caracteriza los nervios de $d$-complejos simpliciales.

Teorema 2 (ver [3], p.65). Un complejo simplicial $\mathcal{M}$ es isomorfo al complejo $\mathcal{N}(K)$ para algún $d$-complejo simplicial $K$ si y solamente si $\mathcal{M}$ satisface

(i) cada arista de $\mathcal{M}$ pertenece a lo más d diferentes caras de $\mathcal{M}$

(ii) cada vértice de $\mathcal{M}$ pertenece a lo más $d+1$ diferentes caras de $\mathcal{M}$

Demostración. Estableceremos primero la necesidad de las condiciones (i) y (ii). Por los lemas 1 y 2 , un vértice $v(F)$ de $\mathcal{N}(K)$ pertenece a tantas facetas $\bar{M}$ de $\mathcal{N}(K)$ como distintas caras básicas $M$ de $K$ están contenidas en la careta $F$ de $K$. Dado que $K$ es un $d$-complejo simplicial, este cantidad es a lo más $d+1$, de este modo la condición (ii) es necesaria.

Por otro lado, sea $v^{\prime}=v\left(F^{\prime}\right)$ y $v^{\prime \prime}=v\left(F^{\prime \prime}\right)$ diferentes vértices de $\mathcal{N}(K)$ los cuales pertenecen a la intersección de las diferentes caretas $\bar{M}_{1}, \ldots, \bar{M}_{q}$ de $\mathcal{N}(K)$. Entonces las diferentes caretas $F^{\prime}$ y $F^{\prime \prime}$ de $K$ comparten las $q$ diferentes caras básicas $M_{1}, \ldots, M_{q}$, ya que $K$ es $d$-dimensional, entonces $q \leq d$, de este modo la condición $(i)$ es necesaria.

Ahora probaremos la suficiencia, para un complejo $M$ satisfaciendo $(i)$ y $(i i)$ se desea obtener un $d$-complejo $K$ tal que $M=\mathcal{N}(K)$. Sin pérdida de generalidad se asume $M$ conexo y por tanto $K$ es conexo. Los vértices de $K$ son de los siguientes tipos

(1) a cada careta $N$ de $M$ se asigna un vértice $V=V(N)$ en $K$

(2) a cada vértice $v$ de $M$ el cual no es una cara básica de $M$, y por $(i)$ pertenece a lo más $d$ caras de $M$, se asigna un vértice $V=V(v)$ de $K$

Las caretas $F=F(v)$ de $K$ están en correspondencia uno a uno con los vértices $v$ de $M$.Si un vértice $v$ es una cara básica de $M$, y si $N_{0}, N_{1}, \ldots, N_{k}$ son todas las caretas de $M$ que contienen $v$, en cuyo caso por $($ ii $) k \leq d$, se asigna a $v$ la careta $F(v)$ de $K$ definida como el $k$-símplice con vértices $V\left(N_{0}\right), V\left(N_{1}\right), \ldots, V\left(N_{k}\right)$. Si $v \in M$ no es una cara básica de $M$, y si $N_{1}, \ldots, N_{k}$ son todas las caretas de $M$ que contienen a $v$, por $(i) k \leq d$ y $v \neq \bigcap_{i=1}^{k} N_{i}$, se define la careta $F(v)$ como el $k$-símplice con vértices $V(v), V\left(N_{1}\right), \ldots, V\left(N_{k}\right)$. Esta construcción implica 
(a) para cada vértice $v \in M$ y cada careta $N \in M, v \in N$ si y solo si $V(N) \in F(v)$

(b) cada vértice $V(v) \in K$ del segundo tipo pertenece a solamente una careta de $K$, particularmente $F(v)$

Ahora se muestra que $M$ es isomórfo al complejo $\mathcal{N}(K)$. Por la construcción de $K$, los vértices de $\mathcal{N}(K)$ están en una natural correspondencia uno a uno con los vértices de $M$. Cada careta de $\mathcal{N}(K)$ es una cara de $M$, en efecto: sea $C$ una careta de $\mathcal{N}(K)$ con vértices $v_{0}, v_{1}, \ldots, v_{p}$ donde $p \geq 1$. Esto implica que las caretas $F_{i}=F\left(v_{i}\right), 0 \leq i \leq p$, de $K$ tienen intersección no vacía, con $V \in F_{0} \cap F_{1} \cap \cdots \cap F_{p}$. Entonces $V$ es un vértice del primer tipo de $K$, ya que cada vértice del segundo tipo pertenece a una careta simple de $K$, luego existe una careta $N$ de $M$ tal que $V=V(N)$. Entonces por $(a), V(N) \in F_{i}=F\left(v_{i}\right)$ es equivalente a $v_{i} \in N$, luego $C$ es una cara de $M$. Así cada careta de $\mathcal{N}(K)$ y cada cara, es también una cara de $M$.

Por otro lado, sea $N=\left(v_{0}, \ldots, v_{r}\right)$ una careta de $M$, el vértice $V(N)$ pertenece a cada una de las caretas $F\left(v_{i}\right), 0 \leq i \leq r$, de $K$, por lo que $\bigcap_{i=0}^{r} F\left(v_{i}\right) \neq \emptyset$, es decir el símplice $\left[v_{0} ; v_{1} ; \ldots ; v_{r}\right]$ pertenece a $\mathcal{N}(K)$, de este modo cada careta de $M$ pertenece a $\mathcal{N}(K)$.

Corolario 3. Cada complejo simplicial $M$ es isomorfo a $\mathcal{N}(K)$ para algún complejo simplicial $K$. Mas aún, $K$ puede ser elegido como un d-complejo, pero no como un complejo de baja dimensión, donde $d=\max \left\{d^{\prime}, d^{\prime \prime}\right\}$, y los números $d^{\prime} y d^{\prime \prime}$ son definidos del siguiente modo: $d^{\prime}$ es el menor entero $k$ tal que cada arista de $M$ está contenido en a lo más $k$ caretas de $M$, $d^{\prime \prime}$ es el menor entero $k$ tal que cada vértice de $M$ está contenido en a lo más $k+1$ caretas de $M$.

\section{Sobre retractos absolutos y retractos de vecindad absoluta}

El siguiente resultado, conocido como el teorema de extensión de Dugundji, establece que todo espacio $V$ débilmente hereditario $\mathcal{S M}$ separable y metrizable es también un $\operatorname{EA}(X)$.

Teorema 4 (ver [2], p.357). Dado $V$ un espacio vectorial normado, separable y no vacío. Para cada espacio $X$, un subespacio cerrado $A$ de $X$ y toda función continua $f: A \rightarrow V$, existe una función continua $F: X \rightarrow V$ tal que $\left.F\right|_{A}=f$ y $F(X) \subset \operatorname{conv}(f(A))$.

Corolario 5 (ver [8], p.154). Para cada espacio metrizable, separable y no vacío $X$ existe un encaje isométrico $h: X \rightarrow C(X ; \mathbb{R})$ tal que

- cada subconjunto $Y$ de $X, h(Y)$ es un subconjunto cerrado de conv $(h(Y))$

- $h(X) \subset\{f \in C(X ; \mathbb{R}) \mid\|f\| \leq \operatorname{diam}(X)\}$

Corolario 6 (ver [2], p.353). Sea $X \neq \emptyset$ un espacio metrizable y separable entonces $X$ se puede encajar en el cubo de Hilbert $\mathcal{C H}=\prod_{i=1}^{\infty}[0 ; 1]_{i}$, donde $[0 ; 1]_{i}$ es una copia de $[0 ; 1]$.

Definición 3.1. Decimos que un espacio metrizable, separable y no vacío $X$ es contráctil, si existe un punto $x_{0} \in X$ y una función continua sobreyectiva $H: X \times[0 ; 1] \rightarrow X$ tal que para todo $x \in X, H((x ; 0))=x$ y $H((x ; 1))=x_{0}$.

Definición 3.2. Sea $X$ un espacio metrizable, separable y no vacío y $A$ un subespacio no vacío de $X$. Decimos que $A$ es un retracto de $X$ si existe una función continua $r: X \rightarrow A$ tal que $r(a)=a$ para todo $a \in A$. La función $r$ es denominada una retracción.

Teorema 7. Sea $X$ un espacio metrizable, separable, no vacio y $A$ un subespacio no vacio de $X$. Si $A$ es un retracto de $X$, entonces $A$ es cerrado en $X$.

Demostración. Ver [8], p.156. 
Teorema 8. Un subespacio $A$ de un espacio metrizable, separable y no vacio $X$ es un retracto de $X$ si y solo si para cada espacio $Y$ y cada función continua $f: A \rightarrow Y$ existe una función continua $F: X \rightarrow Y$ tal que $\left.F\right|_{A}=f$.

Demostración. Ver [8], p.156.

Teorema 9. Todo retracto de un espacio metrizable, separable, no vacío y contráctil es contráctil.

Demostración. Ver [8], p.157.

Definición 3.3. Sea $X$ un espacio metrizable, separable, no vacío y $A$ un subespacio no vacío de $X$. Decimos que $A$ es un retracto de vecindad de $X$ si existe un abierto $U$ de $X$ tal que $A \subset U$ y una retracción $r: U \rightarrow A$.

Definición 3.4. Decimos que un espacio metrizable, separable y no vacío $X$ es un retracto absoluto si para cada espacio $Z$ y cada encaje $h: X \rightarrow Z$ (esto es $X$ y $h(X)$ son homeomorfos o $Z$ contiene una copia de $X)$ tal que $h(X)$ es un subconjunto cerrado de $Z$, se tiene que $h(X)$ es un retracto de $Z$.

Definición 3.5. Decimos que un espacio metrizable, separable y no vacío de $X$ es un retracto de vecindad absoluto si para cada espacio $Z$ y cada encaje $h: X \rightarrow Z$ tal que $h(X)$ es un subconjunto cerrado de $Z$, se tiene que $h(X)$ es un retracto de vecindad de $Z$.

Note que todo retracto absoluto es un retracto de vecindad absoluto, lo recíproco no es cierto. Usando las definiciones tenemos el siguiente resultado.

Teorema 10. El ser un extensor absoluto y un extensor de vecindad absoluto son propiedades topológicas.

Demostración. Ver [8], p.157.

Ahora se probará que los conceptos de retracto absoluto y extensor absoluto son equivalentes, así también los conceptos de retracto de vecindad absoluto y extensor de vecindad absoluto.

Teorema 11. Sea $X$ un espacio metrizable, separable y no vacío. Entonces

(a) $X$ es un retracto de vecindad absoluto si $y$ solo si $X$ es un extensor de vecindad absoluto

(b) $X$ es un retracto absoluto si y solo si $X$ es un extensor absoluto

Demostración. Ver [8], p.157.

Teorema 12. Si $C$ es un subconjunto convexo de un espacio vectorial metrizable, separable y no vacio $V$, entonces $C$ es un retracto absoluto.

Demostración. Sea $Z$ un espacio y $h: C \rightarrow Z$ un encaje tal que $h(C)$ es cerrado en $Z$. Dado que $h^{-1}: h(C) \rightarrow C$ es una función continua y usando el teorema 4, existe una función continua $H: Z \rightarrow \operatorname{conv}(C)$ tal que $\left.H\right|_{h(C)}=h^{-1}$. Como $C$ es convexo, $\operatorname{conv}(C)=C$. Sea $r: Z \rightarrow h(C)$ definida como $r=h \circ H$, entonces $r$ es una función continua y $r(h(c))=h \circ H(h(c))=h \circ$ $h^{-1}(h(c))=h(c)$, de donde $r$ es una retracción. Por tanto $C$ es un retracto absoluto.

Teorema 13 (ver [1] pp. 230). Si K es una realización simplicial de una descomposición regular de un espacio $A$, entonces $A$ es homeomorfo con un retracto de deformación de el politopo $|K|$.

Corolario 14. Si el complejo simplicial $K$ es una realización del nervio de una descomposición regular de un espacio finito dimensional $A$, entonces el espacio $A$ y el politopo $|K|$ tienen el mismo tipo de homotopía. 


\subsection{Aplicaciones en geometría convexa}

\section{Estructura convexa}

Sea $X$ un conjunto no vacío. Una familia $\mathcal{C}$ de subconjuntos de un conjunto $X$ es denominado una convexidad sobre $X$ si

(C-1) $\emptyset, X \in \mathcal{C}$

(C-2) $\emptyset \neq D \subset \mathcal{C}$ entonces $\bigcap D \in \mathcal{C}$ (estable por intersecciones)

(C-3) $\emptyset \neq D \subset \mathcal{C}$ es totalmente ordenado por inclusión, entonces $\bigcup D \in \mathcal{C}$ (estable por uniones anidadas)

El par $(X, \mathcal{C})$ o equivalentemente $\mathcal{C}(X)$ es denominado una estructura convexa. Los elementos de $\mathcal{C}$ son denominados conjuntos convexos y sus complementos conjuntos cóncavos.

Por el axioma (C-1) cualquier subconjunto $A$ de una estructura convexa $\mathcal{C}(X)$ está incluida en al menos un conjunto convexo, esto es $X$.

Por el axioma (C-2) está bien definida la envolvente convexa $\operatorname{conv}(A)$ de un subconjunto $A$ de $\mathcal{C}(X)$

$$
\operatorname{conv}(A)=\bigcap\{C \mid A \subseteq C, C \in \mathcal{C}\}
$$

la cual es el menor conjunto convexo en el que $A$ está contenido.

Por el axioma (C-3) un conjunto es convexo sí y solo si incluye la envolvente de cada uno de sus subconjuntos finitos.

Si $F$ es un conjunto finito, $\operatorname{conv}(F)$ es denominado un politopo.

Sea $(X, \mathcal{C})$ una estructura convexa y sea $Y$ un subconjunto de $X$. La familiade conjuntos $\mathcal{C}_{Y}=\{C \cap Y \mid C \in \mathcal{C}\}$ es una convexidad sobre $Y$, denominada la convexidad relativa de $Y$.

El operador envolvente convexa $\operatorname{conv}_{Y}$ de un espacio $\left(Y, \mathcal{C}_{Y}\right)$ satisface

$$
\forall A \subseteq Y, \operatorname{conv}_{Y}(A)=\operatorname{conv}(A) \cap Y
$$

Definición 3.6. Sea $(X, \mathcal{C})$ una estructura convexa y $\mathcal{T}$ una topología sobre $X$. Se dice que $\mathcal{T}$ es compatible con la estructura convexa $(X, \mathcal{C})$ si todos los politopos de $\mathcal{C}$ son cerrados en $\mathcal{T}$. La terna $(X, \mathcal{T}, \mathcal{C})$ es denominada una estructura convexa topológica.

En $(X, \mathcal{T}, \mathcal{C})$ la colección de conjuntos cerrados en $(X, \mathcal{T})$ son subconjuntos de $\mathcal{C}$.

Definición 3.7. Sea $(X, \mathcal{C})$ un espacio topológico y $\mathcal{C}$ una convexidad sobre $X$. Entonces la terna $(X, \mathcal{T}, \mathcal{C})$ es denominada un espacio topológico convexo.

Teorema 15 (ver [2]). Cualquier conjunto convexo en un espacio topológico lineal localmente convexo es un $\mathrm{EA}(\mathfrak{C})$ para la clase $\mathcal{M}$ de los espacios metrizables.

\section{Invariantes convexos: números de Helly, Caratheodory y Radon}

Los números de Helly, Caratheodory y Radon son centrales en convexidad abstracta. Cada uno de ellos es definido como un grado de independencia tolerado por una estructura convexa, y son invariantes por isomorfismo.

Sea $X$ una estructura convexa y $F \subseteq X$ un conjunto finito no vacío. Se tiene las siguientes definiciones para el conjunto $F$

$$
\text { Helly dependiente: si } \bigcap_{a \in F} \operatorname{conv}(F \backslash\{a\}) \neq \emptyset
$$


Caratheodory dependiente: si $\operatorname{conv}(F) \subseteq \bigcup_{a \in F} \operatorname{conv}(F \backslash\{a\})$

Radon dependiente: si existe una partición $\left\{F_{1}, F_{2}\right\}$ de $F$ tal que $\operatorname{conv}\left(F_{1}\right) \cap \operatorname{conv}\left(F_{2}\right) \neq \emptyset$

De este modo en una estructura convexa $X$ se tiene definido tres números

$h(X) \leq n$ si y solo si cada conjunto finito $F \subseteq X$ con $\# F>n$ es Helly dependiente

$c(X) \leq n$ si y solo si cada conjunto finito $F \subseteq X$ con $\# F>n$ es Caratheodory dependiente

$r(X) \leq n$ si y solo si cada conjunto finito $F \subseteq X$ con $\# F>n$ es Radon dependiente

El siguiente teorema es un resultado clásico para espacios vectoriales reales.

Teorema 16 (ver [4] y [12] p. 168). Sea $V$ un espacio vectorial de dimensión n sobre un campo totalmente ordenado

1. (Teorema de Helly) si $\mathcal{D}$ es una colección finita de conjuntos convexos en $V$, si cualquier subcolección de $n+1$ elementos posee intersección no vacía, entonces los miembros de $\mathcal{D}$ tienen un punto en común. Mas aún, existe alguna subcolección de $n$ elementos que posee intersección vacía.

2. (Teorema de Caratheodory) si $A \subseteq V$ y $p \in \operatorname{conv}(A)$ entonces existe un conjunto $F \subseteq A$ con $\# F \leq n+1$ y $p \in \operatorname{conv}(F)$. Mas aún, existe un conjunto $A$ y un punto $p \in \operatorname{conv}(A)$ tal que $p \notin \operatorname{conv}(F)$ para cada conjunto $F \subseteq A$ con $\# F \leq n$.

3. (Teorema de Radon) si $F \subseteq V$ es un conjunto finito con al menos $n+1$ puntos entonces existe una partición $\left\{F_{1}, F_{2}\right\}$ de $F$ tal que conv $\left(F_{1}\right) \cap \operatorname{conv}\left(F_{2}\right) \neq \emptyset$. Si $\# F \leq n+1$ entonces dicha partición no existe.

El teorema de Helly asegura que el número de Helly de una familia de conjuntos convexos, en un espacio vectorial de dimensión $n$, es $d+1$.

Teorema 17. Si r, h, c son respectivamente los números de Radón, Helly y Caratheodory de un espacio de convexidad, entonces

$$
h+1 \leq r \leq c(h-1)+2
$$

La cota inferior es debida a Levi [7], y es alcanzado para un gran número de espacios de convexidad. La cota superior tiene a ser obtenida por diversos autores, una prueba se puede encontrar en Sierksma [10].

Un conjunto $X \subset \mathbb{R}^{d}$ es $(m ; k)$-divisible si este puede ser partido en $m$ subconjuntos cuyas envolventes convexas se intersecan en un conjunto de dimensión al menos $k$, considerando $m \geq 2$ y $0 \leq k \leq d$. Un conjunto $(m ; 0)$ divisible es también llamado $m$-divisible.

Consideremos el par $(S, \mathcal{C})$, el número $r_{m}(S, \mathcal{C})$ para cada $m \geq 2$, es el menor número $r_{m}$ tal que cualquier conjunto de $r_{m}$ puntos en $S$ es $m$-divisible. Este número, si es que existe, es denominado el $m$-ésimo número de Tverberg de $(S, \mathcal{C})$. En particular $r_{2}$ es el número de Radón.

La determinación de los números de Tverberg es difícil y no se ha logrado mucho al respecto. Un resultado conocido es el siguiente: si $r_{2}$ es finito, entonces $r_{m}$ es finito para todo $m$ ([6], p. 124).

Sea $\gamma(d ; m ; k)$ el menor entero $\gamma$ con la propiedad que cada conjunto de al menos $\gamma$ puntos en $\mathbb{R}^{d}$ es $(m ; k)$-divisible. 
Teorema 18 (Birch-Tverberg [11]).

$$
\gamma(d ; m ; 0)=(m-1)(d+1)+1
$$

Cuando $m=2$ se tiene el teorema de Radón. La mejor cota superior obtenida para $r_{m}$ en términos de $m$ y $r_{2}$ en un espacio de convexidad es

$$
r_{m}=(m-1)\left(r_{2}-1\right)+1
$$

Existen especulaciones en saber si

$$
r_{m} \leq(m-1)\left(r_{2}-1\right)+1
$$

se tiene para cualquier $m$ y cualquier espacio de convexidad con número de Radón $r_{2}$. De conseguirse, sería equivalente a dar una prueba puramente combinatoria del teorema de Tverberg.

\section{Un resultado en topología algebraica y geometría convexa}

El nervio de una colección $\mathcal{C}$ de subconjuntos de un conjunto $X$ representa el patrón de intersección de los componentes de $\mathcal{C}$. En el corolario 14 se relacionó a un espacio finito dimensional y un politopo que proviene de la realización del nervio de la descomposición del espacio finito, esto puede extenderse a un resultado general.

Un complejo simplicial $K$ es $d$-representable si es isomorfo al nervio de una colección finita de conjuntos convexos en $\mathbb{R}^{d}$. A dicha colección de conjuntos convexos se denomina una $d$ representación de $K$. De este modo, los complejos $d$-representables registran todos los patrones de intersección de una colección finita de conjuntos convexos en $\mathbb{R}^{d}$.

Usando la noción de $d$-representabilidad, el teorema de Helly puede ser reformulado: un complejo simplicial $d$-representable no contiene un agujero simplicial $k$-dimensional para $k \geq d$.

En la definición de $d$-representabilidad, es posible tomar varias restricciones en los conjuntos convexos sin afectar la definición. Estas restricciones son resumidas en el siguiente lema.

Lema 3. Sea $K$ un complejo simplicial. Las siguientes condiciones son equivalentes

1. $K$ es d-representable

2. K es el nervio de una colección de politopos convexos

3. $K$ es el nervio de una colección de conjuntos convexos compactos

4. K es el nervio de una colección de conjuntos convexos abiertos acotados

Demostración. $(1) \Rightarrow(2)$. Sea $\left\{C_{v}\right\}$ una $d$-representación de $K$. Para una cara $\alpha$ de $K$ considere el punto $p_{\alpha}$ perteneciente a todos $\operatorname{los} C_{v}$, donde $v \in \alpha$. Para $v \in V(K)$ sea $C_{v}^{\prime}=\operatorname{conv}\left\{p_{\alpha}: v \in \alpha\right\}$, entonces $\left\{C_{v}^{\prime}\right\}$ es una representación de $K$ mediante politopos convexos, con $\left\{C_{v}^{\prime}\right\} \subseteq\left\{C_{v}\right\}$.

Las demás implicaciones son inmediatas.

Existen otras dos importantes clases de complejos simpliciales vinculados a $d$-representabilidad. De un modo informal: (i) un complejo simplicial es $d$-colapsable si este puede ser desvanecido removiendo caras de dimensión a lo más $d-1$ los cuales están contenidos en una sola careta, (ii) un complejo simplicial es $d$-Leray si sus subcomplejos inducidos no contienen, homológicamente, agujeros $d$-dimensionales.

Wegner [13] prueba que complejos simpliciales $d$-representables son $d$-colapsables y también que complejos $d$-colapsables son $d$-Leray.

Teorema 19 (Wegner-Perelman). Sea $K$ un complejo simplicial d-dimensional. Entonces $K$ es $(2 d+1)$-representable. 


\section{Conclusión y recomendaciones}

El dividir un espacio topológico en piezas como los complejos simpliciales, no siempre es posible. Y las caracterizaciones de cuando ello es posible son necesarias en aras de encaminar métodos usando complejos simpliciales. Los extensores absolutos y los extensores de vecindad absolutos dan un medio para extender subespacios cerrados al espacio total. Sin embargo el sentido inverso, es decir cuando un espacio es reducible en un subespacio cerrado, es mas conveniente a fin de reducir las dimensiones necesarias para encontrar una realización geométrica de un complejo simplicial que identifique a un espacio topológico determinado.

Tanto el nervio de un cubrimiento como el nervio de un complejo simplicial resumen características del patrón de intersección de los conjuntos que la componen. En particular si estos son convexos y se encuentran en un espacio metrizable dan lugar a dimensiones específicas de la $d$-representabilidad de un complejo simplicial.

Extender los resultados de las caracterizaciones del patrón de intersecciones para conjuntos no convexos es un aspecto del

Desde el ámbito computacional, se sabe que en general para $d \geq 5$ es algorítmicamente indecible cuando un complejo simplicial es $d$-representable. Sin embargo existe un algoritmo polinomial que permite comprobar si un complejo simplicial de dimensión $d / 2$ es embebible en $\mathbb{R}^{d}$, para $d \geq 6$ par o $d=2$. Para mayor referencia ver [9]. 


\section{Referencias bibliográficas}

[1] Borsuk, K. (1948). On the imbedding of systems of compacta in simplicial complexes. Fundamenta Mathematicae 35, 217-234.

[2] Dugundji, J. (1951). An extension of Tietze's theorem. Pacific J. Math. 1(3), 353-367.

[3] Grünbaum, B. (1970). Nerves of simplicial complexes. Aeq. Math. 4, 63-73.

[4] Helly, E. (1923). Über Mengen konvexer Körper mit gemeinschaftlichen Punkten. Jahresber. Deutsch. Math. Verein. 32, 175-176.

[5] Hu, S. (1965). Theory of Retracts. Wayne State University Press, Detroit.

[6] Jamison-Waldner, R. (1981). Partition numbers for trees and ordered sets. Pacific J. Math. 96(1), 115-140.

[7] Levi, F. (1951). On Helly's theorem and the axioms of convexity. J. Indian Math. Soc. 15, 65-76.

[8] Macias, S. (2013). Una introducción a los retractos absolutos y a los retractos de vecindad absolutos. Revista Integración Escuela de Matemáticas, Universidad Industrial de Santander $31(2), 153-164$.

[9] Matousek, J., Tancer, M., Wagner, U. (2009). Hardness of embedding simplicial complexes in $\mathbb{R}^{d}$. J. Eur. Math. Soc. 13(2), 259-295.

[10] Sierksma, G. (1977). Relationships between Caratheodory, Helly, Radon and exchange numbers of convexity spaces. Nieuw Arch. Wisk., 25(3), 115-132.

[11] Tverberg, H. (1966). A generalization of Radon's theorem. Journal of the London Mathematical Society, 41(1), 123-128.

[12] Van de vel, M. (1993). Theory of Convex Structures. Elsevier Science.

[13] Wegner, G. (1975). d-collapsing and nerves of families of convex sets. Arch. Math., 26, 317321. 